\title{
Spatial working memory load impairs signal enhancement, but not attentional orienting
}

\author{
Suk Won Han ANd Min-ShIK Kim \\ Yonsei University, Seoul, Korea
}

\begin{abstract}
The present study examined the effect of spatial working memory load on the attentional cuing effect. It is well-known that spatial working memory and spatial attention functionally overlap or share a common resource. Given this functional overlapping, it is possible that spatial working memory would also interact with the attentional cuing effect. Considering the distinction between channel selection and channel enhancement by attention (Prinzmetal, McCool, \& Park, 2005), we expected that the interaction between spatial working memory and the cuing effect would differ with attentional processing type. Two experiments conducted with the spatial cuing paradigm showed that the magnitude of the cuing effect as measured by reaction time, reflecting channel selection, was uninfluenced by spatial working memory load. In contrast, spatial working memory load reduced the cuing effect as measured by accuracy, reflecting channel enhancement. These results suggest that spatial working memory load impairs signal enhancement by attention and does not influence attentional orienting per se. The interaction between spatial working memory and visual perception is also discussed.
\end{abstract}

The visual system cannot process all the sensory inputs that it receives, because our capacity for processing visual information is limited. When faced with a huge amount of information, our selective attention allocates limited resources to the most critical and relevant information, thus preventing information overload. By attending to a specific component of a visual scene, selective attention allows certain information to be processed in finer detail or given greater processing priority.

It has been suggested that visual or spatial information is maintained in working memory via allocations of attention to memory representations, activated online, of relevant objects or locations. In response, numerous studies have investigated selective attention together with working memory (Awh, Jonides, \& Reuter-Lorenz, 1998; Cowan, 2001; Engle, 2002). A number of studies have proposed that spatial selective attention and spatial working memory functionally overlap and that spatial attention plays a critical role in the maintenance of spatial information in working memory (Awh \& Jonides, 2001; Awh et al., 1998; Jonides, Lacey, \& Nee, 2005). Awh et al. (1998) reported impaired spatial working memory accuracy with shifts of attention from a memorized location. They concluded that memorizing locations requires selective attention to be shifted to those locations. In addition, a recent brain imaging study has demonstrated considerable overlap in areas of the brain active during selective attention and spatial working memory tasks (Corbetta, Kincade, \& Shulman, 2002). Oh and Kim (2004) and Woodman and Luck (2004) noted that the maintenance of spatial information in working memory impairs the efficiency of concurrent visual search tasks. Their studies imply that spatial working memory and visual search share a common resource.

This close interaction between spatial working memory and selective attention needs to be specified further, because attentional effects on visual processing are heterogeneous. Prinzmetal and colleagues suggested that attention affects visual processing via two distinct mechanisms (Prinzmetal, McCool, \& Park, 2005). First, attention can enhance the perceptual representation of an attended location (Luck, Hillyard, Mouloua, \& Hawkins, 1996; Luck \& Thomas, 1999; Moore \& Egeth, 1998). In data-limited conditions (Norman \& Bobrow, 1975), in which stimuli are presented briefly, observers respond faster and more accurately when a spatial cue is given. In this case, the enhanced performance afforded by the cue is mediated by sensory modulation on the attended location. That is, the primary factor that improves behavioral performance under data-limited conditions is the quality of the perceptual representations of stimuli. Prinzmetal et al. have labeled this processing channel enhancement and suggest that it corresponds with what other researchers have termed signal enhancement (Carrasco, Williams, \& Yeshurun, 2002; Lu \& Dosher, 1998; Yeshurun \& Carrasco, 1999). Generally, in data-limited conditions, response accuracy has been used as a primary dependent variable for measuring behavioral performance, because the quality of visual information is degraded and observers are required to take enough time to respond as accurately as possible. The point to be noted is that an attentional cuing effect is found in accuracy only if the perceptual representation is enhanced. Second, at-

M.-S. Kim, kimm@yonsei.ac.kr 
tention can affect behavioral performance without sensory modulation on the attended location. Upon receiving a spatial cue, participants select the cued location for processing prior to an uncued location. This prioritization, generally revealed by the benefit in reaction time, is called channel selection. This kind of selection has been investigated under data-unlimited conditions, in which stimuli are presented long enough. In this resource-limited, rather than data-limited, condition, the attentional cuing effect is usually measured in terms of reaction time, because participants show nearly perfect accuracy.

The distinction between channel selection and channel enhancement has been proposed in other studies (Moore \& Egeth, 1998; Mordkoff \& Egeth, 1993; Santee \& Egeth, 1982). It has often been suggested that channel enhancement reflects an attentional effect on visual processing in early, perceptual stages of processing and that channel selection shows an attentional effect on late, postperceptual processing (Prinzmetal et al., 2005). We cannot be sure whether these two forms of processing would be separated by the time course. However, channel enhancement could be entirely separate from channel selection because the latter could consist of selection for a motor response or memory and the former could consist of selection for enhanced perceptual processing.

Given the distinction between channel selection and channel enhancement, working memory load could influence the attentional cuing effect in two different ways. On the one hand, working memory load could impair the efficient allocation of attention, reducing any type of attentional cuing effect. On the other hand, it is possible that working memory would interact with attention in different ways, depending on the type of attentional processing. To the best of our knowledge, no other studies have resolved such a question. A recent brain imaging study by Postle and colleagues has shown that, although brain activation areas overlapped considerably during selective attention and spatial working memory tasks, they were not identical (Postle, Awh, Jonides, Smith, \& D’Esposito, 2004). In that study, the frontal eye field was activated during selective attention but was not activated during the spatial working memory task. This suggests that not every type of attentional processing is influenced by spatial working memory, because the frontal eye field is known to be critical for attentional orienting during spatial cuing and visual search tasks (Hamker, 2004; Serences \& Yantis, 2006).

In the present study, the spatial cuing paradigm was used to investigate whether spatial working memory load could influence the attentional cuing effect. Concerning this issue, Jonides (1981) showed that working memory load exceeding the capacity limit reduced the effect of endogenous attention. Jonides, however, did not examine which type of attentional processing interacted with working memory load because he did not study cuing effects in terms of reaction time and accuracy separately. In another study, a secondary task eliminated attentional capture by introducing a transient event (a new object onset) in a visual search task (Boot, Brockmole, \& Simons, 2005). This result suggests that executive working memory load impairs exogenous orienting, but a more specific influence of working memory on attention has not yet been investigated.

In our experiments, either a transient cue (peripheral cue) predicting the target location or a noninformative central cue (neutral cue) was given, and participants had to discriminate which side of a Landolt-square target had a gap. Participants were required to perform this visual acuity task in either the presence or the absence of spatial working memory load. We studied the cuing effect by measuring reaction time and accuracy in separate experiments. As already mentioned, enhancements to accuracy under data-limited conditions reflect channel enhancement. On the other hand, reaction time benefits without any advantage in accuracy under data-unlimited conditions are mediated by channel selection. The present study aimed to determine whether the effect of spatial working memory load on the cuing effect could differ depending on the attentional processing type. Specifically, we expected that spatial working memory load would not influence the magnitude of the cuing benefit as measured by reaction time. This supposition is rooted in the findings of Postle et al. (2004), who showed that attentional selection and spatial working memory may be mediated by separate mechanisms. In contrast, the cuing effect as measured by accuracy would be reduced by spatial working memory load, meaning that spatial working memory load would impair signal enhancement (orienting for enhanced perceptual processing) without influencing other attentional orienting. The interference of spatial working memory on the selective attention task (Oh \& Kim, 2004; Woodman \& Luck, 2004) might have been observed because channel enhancement, rather than channel selection, had been impaired by spatial working memory.

\section{EXPERIMENT 1}

Experiment 1 was designed to examine whether spatial working memory load would influence the magnitude of the spatial cuing effect as measured by reaction time. To maximize the effect of spatial working memory load, participants were required to memorize four locations. We reasoned that memorizing four locations would be difficult enough to exert a load effect, because Vogel and colleagues suggested four objects to be the capacity limit of visual working memory (Vogel, Woodman, \& Luck, 2001). Also, in order to maximize the cuing benefit, we designed a concurrent visual acuity task (Landolt-square discrimination) to be perceptually demanding, using a relatively large eccentricity and small gap size.

\section{Method}

Participants. Fourteen undergraduate students at Yonsei University participated for course credit. All had normal or corrected-tonormal vision and were naive as to the purpose of the experiment. Informed consent was obtained from each of the participants.

Stimuli and Apparatus. The stimuli were presented on an LG 17-in. CRT monitor with a black background. The monitor refresh rate was $75 \mathrm{~Hz}$, and the resolution was set to $1,024 \times 768$ pixels. The experiment was programmed using MATLAB equipped with the Psychophysics Toolbox (Brainard, 1997) and was run on an IBM-compatible computer with a Pentium 4 processor. The viewing 
distance was set at $57 \mathrm{~cm}$, using an adjustable chinrest. Participants were required to fixate a small gray $\operatorname{dot}\left(0.3^{\circ} \times 0.3^{\circ}\right.$ in visual angle $)$ throughout the experiment. The memory array comprised four white squares (each $0.43^{\circ} \times 0.43^{\circ}$ ) presented at four locations chosen randomly from eight locations spaced evenly on an imaginary circle with a radius of $2.5^{\circ}$. The target was a Landolt-square $\left(1^{\circ} \times 1^{\circ}\right)$, with a gap $\left(0.07^{\circ} \times 0.11^{\circ}\right)$ on its left or right side and a line thickness of $0.07^{\circ}$. The target was presented at one of eight locations spaced evenly on an imaginary circle with a radius of $7.5^{\circ}$. The target appeared equally often with the left side gap and with the right side gap in randomized order. On half of the trials, a green horizontal bar $\left(0.64^{\circ} \times 0.28^{\circ}\right)$ was presented as a peripheral cue $1^{\circ}$ above the top of the target, preventing forward masking (Carrasco et al., 2002). On the other half, a green circle with a diameter of $0.39^{\circ}$ was presented at the center of the screen. At the end of each trial, a single square identical to a memory stimulus was presented. The square was presented either at one of the locations occupied by the memory array or at a location not occupied by memory stimuli, equally often with randomized order.

Procedure and Design. There were dual- and single-task conditions, which were blocked. A dual-task condition trial sequence is shown in Figure 1. At the beginning of each trial, "ABCD" was presented at the center of the screen for $506 \mathrm{msec}$, which prompted participants to begin verbal rehearsal, repeating "ABCD.” Verbal rehearsal, repeating "ABCD," was emphasized in both conditions.

In the dual-task condition, a fixation dot remained present throughout each experiment. Following presentation of the fixation dot for $1,000 \mathrm{msec}$, the memory array appeared for $506 \mathrm{msec}$. The participants were required to memorize the location of each memory square. After each 506-msec interval, a peripheral cue or a neutral cue was presented for $40 \mathrm{msec}$. The offset of the cue was followed by a $93-$ msec presentation of a blank screen and then the presentation of the target. The participants were required to make a speeded response, and the target was present until they responded. If participants did not respond within $1,500 \mathrm{msec}$, an error sound was heard, and the trial was removed from analysis. At the end of the trial, a single square was presented as a memory test probe. Participants had to judge whether the location of the probe matched the locations occupied by the memory array.
In the single-task condition, the memory array was replaced by a blank screen, and the memory test probe was replaced by a blank screen for $1 \mathrm{sec}$. All other settings were identical to those of the dual-task condition.

Participants each performed three blocks of dual- and single-task trials; the order of task conditions was counterbalanced across participants. Thus a single block consisted of 64 trials, and 192 trials were assigned to each task condition (a total of 384 trials).

\section{Results and Discussion}

The overall accuracy rate of target responses was $98.7 \%$ (98.6\% for valid and 99\% for neutral trials in single-task condition, and $99 \%$ and $98.4 \%$ in the dual-task condition), and the memory accuracy rate was $71.5 \%(70.6 \%$ for valid and $72.3 \%$ for neutral trials). These did not differ by task condition or cue type. The reaction time results from Experiment 1 are shown in Figure 2. The trials with correct target responses were included in the analysis of reaction times. The reaction times for correct target responses were analyzed in all conditions. In the dual-task condition, only trials with correct target and memory responses were included in the analysis. Median reaction times of participants were analyzed in a within-subjects two-way ANOVA with task condition (single task, dual task) and cue (peripheral cue, neutral cue) as factors. The main effects of both task condition and cue were significant $[F(1,13)=4.69, p<.05$, and $F(1,13)=209.01, p<$ .001 , respectively]. The interaction of cue $\times$ task was not significant $(F<1)$.

As expected, participants responded faster to spatial cues than to neutral cues. Spatial working memory load increased the reaction time to complete the visual acuity task, indicating that working memory load negatively affected concurrent visual task performance. The addi-

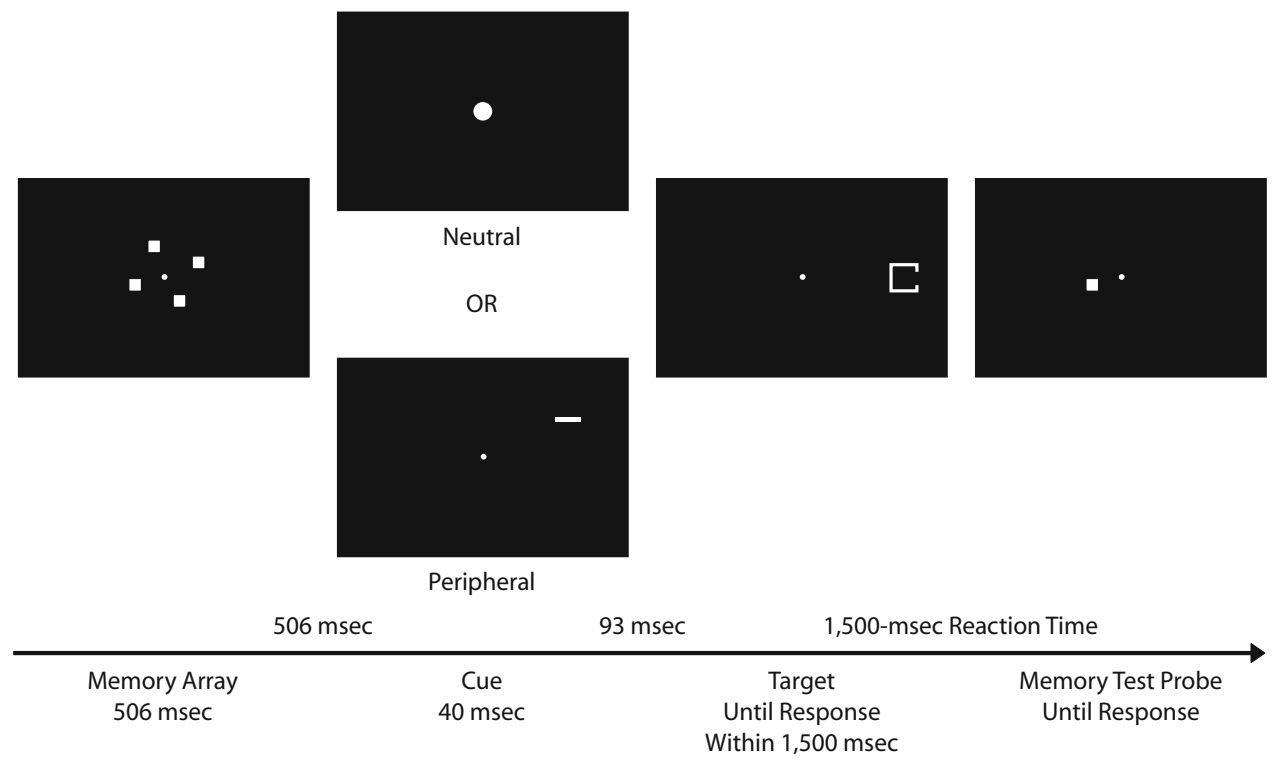

Figure 1. An example of a dual-task trial in Experiment 1. In the dual-task condition, participants had to perform a visual acuity task, memorizing four locations, and a memory test probe was given at the end of each trial. In the single-task condition, blank screens were presented instead of a memory array and memory probe. 


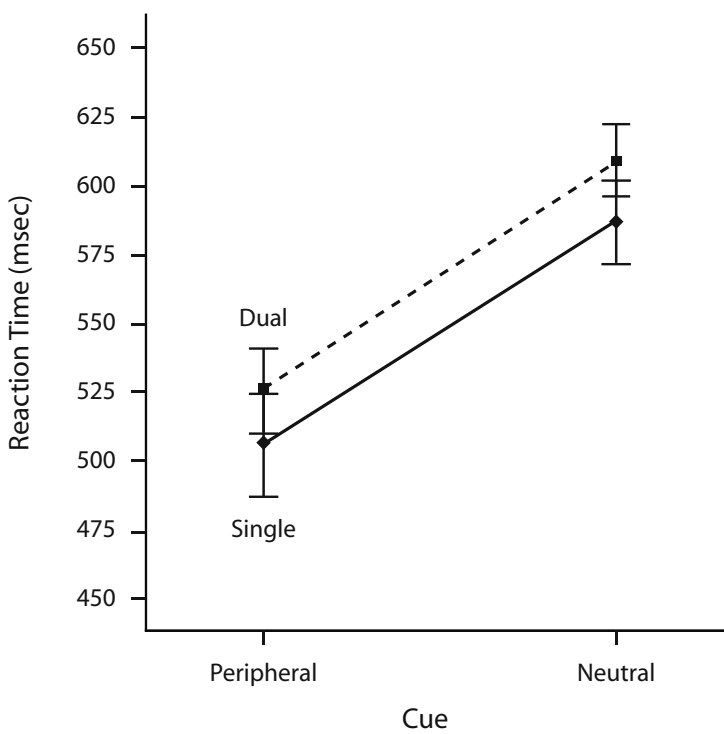

Figure 2. Reaction time results from Experiment 1. Error bars represent standard errors.

tive increase in reaction time in the presence of working memory load might reflect the cost of task switching or delayed response selection (Woodman, Vogel, \& Luck, 2001). However, we did not find any evidence of interaction between working memory load and the attentional cuing effect. Spatial working memory load did not affect the cuing effect as measured by reaction time.

This result showed that attentional orienting was not disrupted by spatial working memory load. That is, while memorizing four locations, participants were able to detect and perceive the transient cue and allocate attention to the cued location without interference from working memory load. This implies that spatial working memory and attentional orienting could be mediated by separate mechanisms.

It is possible that spatial working memory load would influence another type of attentional processing without impeding attentional orienting. Specifically, if spatial working memory load impaired only channel enhancement and not channel selection, then the cuing effect measured by reaction time would not be influenced by spatial working memory load, as in the present experiment. In contrast, the cuing effect measured by accuracy would be decreased. According to Oh and Kim (2004) and Woodman and Luck (2004), spatial working memory load may decrease the efficiency of a concurrent visual search task. The results of the present experiment suggest that decreases in visual search task efficiency might not be due to interference with attention allocation by working memory load. During the trials, participants could have allocated attention to each item serially without impairment, even in the presence of working memory load. However, it would have taken longer for them to process each stimulus, because the attentional benefit in perception would have been reduced by working memory load, perhaps resulting in decreased visual search task efficiency. Experiment 2 was designed to examine whether spatial working memory load would impair channel enhancement.

\section{EXPERIMENT 2}

In Experiment 1, there was no interaction between spatial working memory load and the spatial cuing effect. However, it remained unresolved whether spatial working memory might influence cuing benefit. Attention can not only decrease reaction time in behavioral performance, but also improve the perception of visual stimuli. In Experiment 1 , it was observed that spatial working memory load did not change the magnitude of the attentional benefit as measured by reaction time. Experiment 2 was designed to investigate whether spatial working memory load would influence the magnitude of the spatial cuing effect as measured by accuracy under data-limited conditions .

\section{Method}

Participants. Twelve undergraduate students at Yonsei University participated for course credit. All had normal or corrected-tonormal vision and were naive as to the purpose of the experiment. Informed consent was obtained from each participant.

Stimuli and Apparatus. The stimuli and apparatus were identical to those in Experiment 1, except that a gray rectangle $\left(1.7^{\circ} \times\right.$ $1.5^{\circ}$ ) was used as a mask for the target.

Procedure and Design. The procedure and design were identical to those of Experiment 1, with the following exceptions. A staircase procedure was used to adjust the target duration for each participant to yield an accuracy rate of $65 \%-70 \%$ in the visual acuity task. The target duration ranged from $80 \mathrm{msec}$ to $147 \mathrm{msec}$, after which the mask was presented for $200 \mathrm{msec}$.

\section{Results and Discussion}

Three participants were excluded from the analysis because their overall accuracy rates in the visual acuity task were less than $62 \%$ and their performance on trials with a neutral cue was at the chance level in both the singleand dual-task conditions. Overall memory accuracy was $68 \%$. The accuracy and reaction time results from Experiment 2 are shown in Figure 3. Trials with correct target responses and memory responses were included in the analysis. The overall accuracy rate for the visual acuity task was $70 \%$. Accuracy rates were analyzed as a primary dependent variable, and reaction times were analyzed as a secondary dependent variable, as in Experiment 1. A two-way ANOVA for accuracy showed that both the task and the cue had significant main effects $[F(1,8)=5.67$, $p<.05$, and $F(1,8)=108.33, p<.001$, respectively]. Remarkably, the interaction of task $\times$ cue was significant $[F(1,8)=10.12, p<.05]$, showing that spatial working memory load reduced the attentional cuing effect. The reaction time results were consistent with those for Experiment 1; the significant main effects of task and cue did not interact.

As in Experiment 1, behavioral performance was improved by a spatial cue, and spatial working memory load interfered with the visual acuity task. In Experiment 1, there was no interaction between the attentional cuing effect and spatial working memory load. In contrast, in Experiment 2, spatial working memory load reduced the cuing effect, as measured by accuracy. There was no in- 

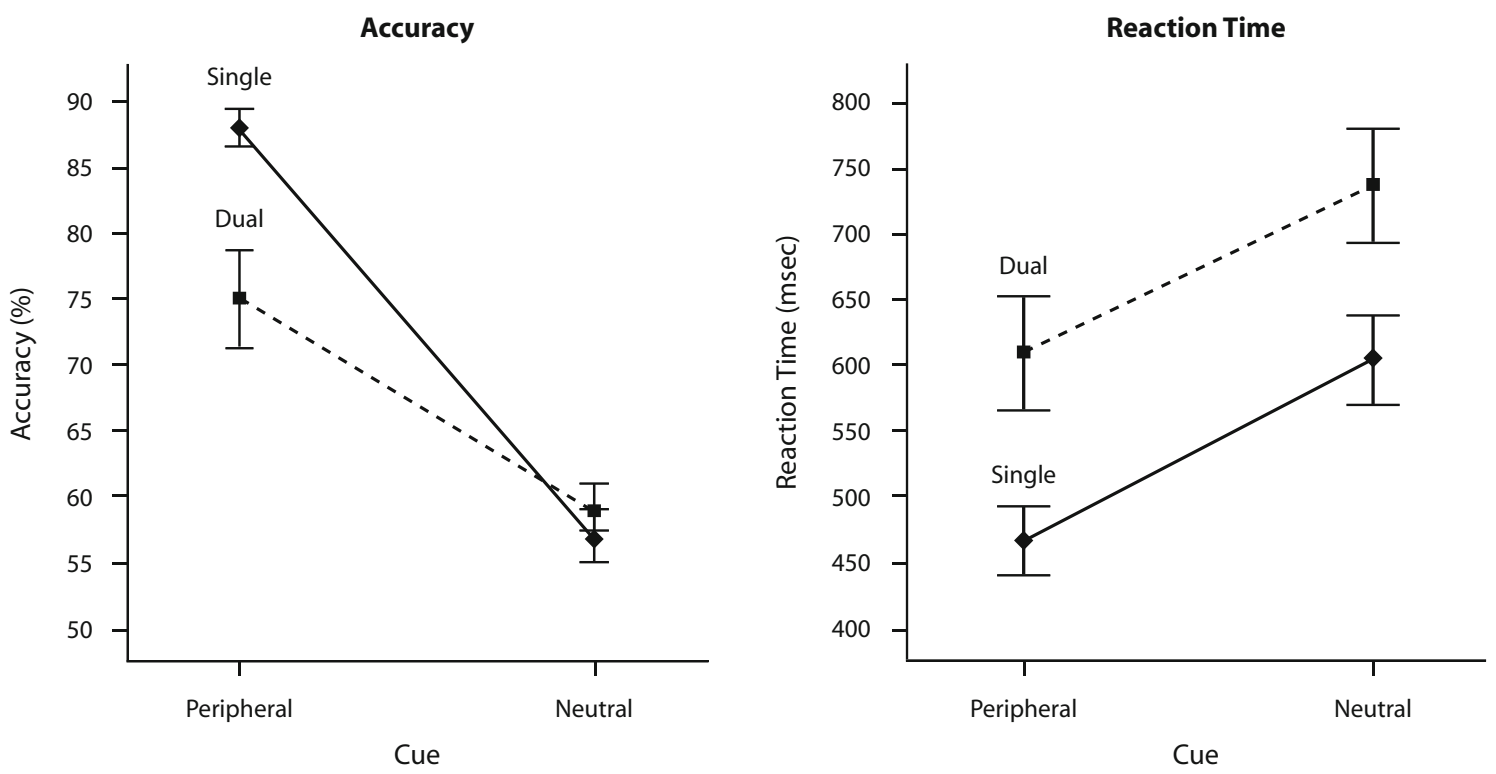

Figure 3. Accuracy and reaction time results from Experiment 2. Error bars represent standard errors.

teraction between spatial working memory load and the cuing effect, as measured by reaction time, in Experiment 2, as in Experiment 1.

However, these data should be interpreted with considerable caution. When a neutral cue was given, accuracy rates for the visual acuity task were $57 \%$ and $59 \%$ for the single- and dual-task conditions, respectively. Although these accuracies exceeded chance, the interaction observed in Experiment 2 could possibly have been caused by a flooring effect. That is, the interaction might have been induced because the accuracy rate of the neutral trials could not be lowered below chance. To reject this alternative, we designed another experiment with a decreased eccentricity of the target location. In Experiments 1 and 2, the target was presented with an eccentricity of $7.5^{\circ}$, which made the visual acuity task demanding. In this experiment, the eccentricity was decreased to $5.5^{\circ}$ in order to improve overall performance. Eleven undergraduate students, naive to the purposes of the experiment, participated. All settings besides the decreased eccentricity were identical to those in the prior experiment.

The accuracy result from the decreased eccentricity experiment is shown in Figure 4. The overall accuracy rate in the visual acuity task was $74 \%$, and the accuracy rates in the neutral cue trials improved to $68 \%$ and $67 \%$ in the single- and dual-task conditions, respectively. The decreased eccentricity elevated the performance level only in the neutral cue trials. This pattern is consistent with the result of a previous study, in which eccentricity had a more pronounced effect on neutral trials than on cued trials (Yeshurun \& Carrasco, 1999). The significant interaction between working memory and the cuing effect was observed again $[F(1,10)=22.54, p<.01]$. The main effect of cue was significant $[F(1,10)=39.89, p<.01]$, and the difference in accuracy for each task condition was also significant $[F(1,10)=9.12, p<.05]$. The reaction time analysis showed significant main effects of cue and task without any interaction between them.

Eye movement control is another important point to be considered. Participants were required to fixate the center of the screen throughout a whole experiment, and the cue stimulus onset asynchrony $(133 \mathrm{msec})$ and target durations (80-147 msec) were brief enough to preclude goal-directed saccades. In many cases, however, the intervals between cue onset and target offset were longer than $250 \mathrm{msec}$, which was long enough for goal-directed saccades to occur (Carrasco et al., 2002; Mayfrank, Kimmig, \& Fischer, 1987; Yeshurun \& Carrasco, 1999). In Experiment 2, with the larger eccentricity, the target durations of all but 1 of the participants exceeded $120 \mathrm{msec}$, and, in the other experiment, with the smaller eccentricity, the target durations of only 5 of the 11 participants were longer than $120 \mathrm{msec}$. Even if eye movements could occur, this cannot be an alternative explanation for the present results. Eye movements could occur in both the singleand dual-task conditions, but accuracy was higher in the single-task condition than in the dual-task condition on the peripheral cue trials in both the experiment with large eccentricity $[F(1,8)=13.43, p<.01]$ and the experiment with small eccentricity $[F(1,10)=20.61, p<.01]$. In contrast, there was no difference between task conditions on the neutral cue trials. This difference cannot be explained by eye movements, so it must be explained by the attentional effect. However, it was also possible that working memory load interfered with eye movements in the dualtask condition (Pearson \& Sahraie, 2003). To confirm that the present result was not confounded by eye movements, the results of 6 participants from the small eccentricity ex- 


\section{Large Eccentricity}

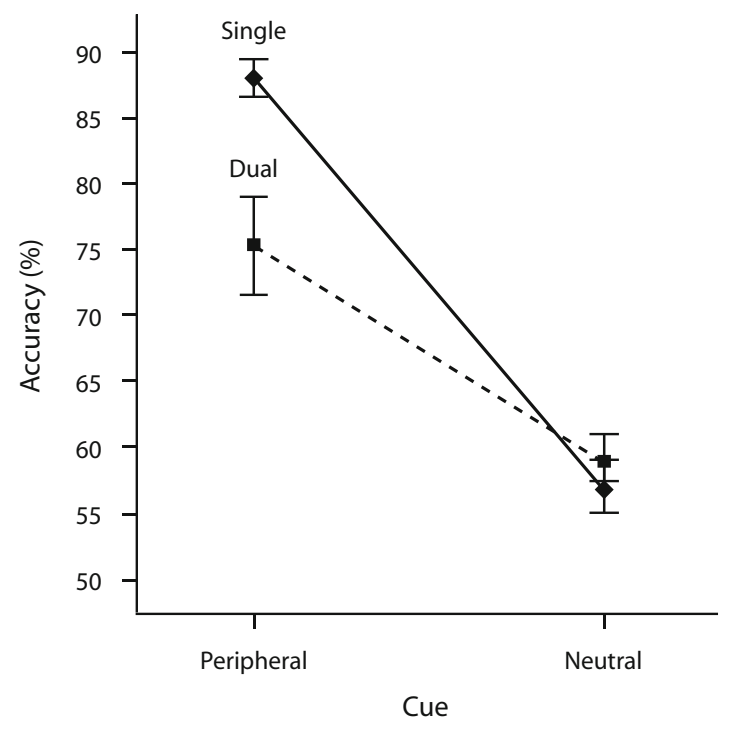

Small Eccentricity

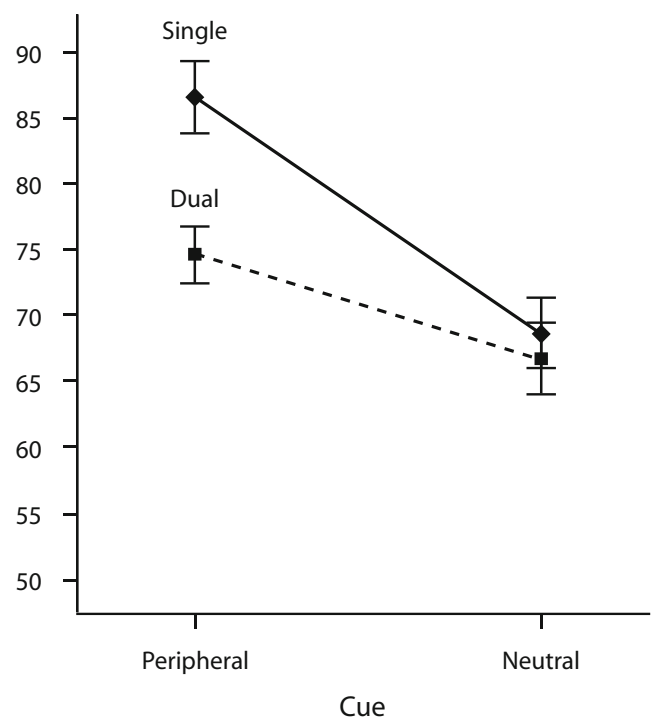

Figure 4. Comparison between the experiment with the large eccentricity and the experiment with the small eccentricity. Error bars represent standard errors.

periment with target durations of less than $107 \mathrm{msec}$ were analyzed. In this case, the intervals between the cue onset and the target offset were shorter than $240 \mathrm{msec}$, precluding goal-directed saccades. The interaction between cue and task condition was significant $[F(1,5)=13.85, p<$ $.05]$, and their patterns were not qualitatively different from those of rest.

In Experiment 2, the decreased cuing effect in the dualtask condition was not induced by disrupted detection of the spatial cue or interference with attentional allocation. Only signal enhancement by attention was impaired while other types of attentional selection remained intact. If spatial working memory load interfered with attentional orienting itself, then the cuing effect as measured by reaction time would also have been reduced. As shown in Experiments 1 and 2, however, the cuing effect, as measured by reaction time, was static in the presence of spatial working memory load.

It should be noted that the attentional advantage in accuracy could be mediated not only by enhanced perceptual representation of the attended location, but also by reduction of location uncertainty by a spatial cue (Luck et al., 1996; Luck \& Thomas, 1999; Prinzmetal et al., 2005). In the present study, the enhanced perceptual representation was a primary factor affecting accuracy, because the transient cue always predicted the target location and the local mask was used to cover the target, which eliminated the uncertainty related to the target location. Although we suggest that signal enhancement is a main mechanism in enhanced perception in our experimental situation because we used the display with the minimal level of external noise, any type of noise reduction or distractor suppression might not be excluded absolutely. The point here is that the cuing effect observed in the present study was mediated mainly by enhanced perceptual representations of stimuli.
The results of these experiments suggest that spatial working memory load affects distinct attentional processing in several different ways. Spatial working memory load did not influence the cuing effect, as measured by reaction time, but it did reduce the attentional advantage measured in terms of accuracy under data-limited conditions. This implies that spatial working memory load does not impair channel selection but rather channel enhancement by attention. The decreases in visual search task efficiency by spatial working memory reported by $\mathrm{Oh}$ and Kim (2004) and Woodman and Luck (2004) could have been induced by a reduced attentional benefit in perception, but not by a disruption of attentional orienting itself.

\section{GENERAL DISCUSSION}

This study investigated the interaction between working memory and attention, in line with numerous previous studies (Awh et al., 1998; Oh \& Kim, 2004; Woodman \& Luck, 2004; Woodman et al., 2001). The present results show that spatial working memory load does not have any effect on attentional benefit in terms of reaction time. In contrast, spatial working memory load reduced the spatial cuing effect, as measured by accuracy, in a data-limited condition, in which stimuli were presented briefly. This result is consistent with the distinction of Prinzmetal et al. (2005), who suggested that attentional benefits to reaction time would be the effect of channel selection and that attentional benefits to accuracy could be mediated by channel enhancement. In our experiments, different patterns of interaction between the attentional effect and working memory were observed, depending on the type of attentional processing. By showing this selective interaction, we reconfirmed the distinction between channel selection and channel enhancement. 
Our results not only are consistent with previous findings of an interaction between spatial working memory and selective attention (Oh \& Kim, 2004; Woodman \& Luck, 2004), but also suggest a refined explanation for prior results. In past experiments, participants had to perform a visual search task, with or without maintaining four locations in working memory. Visual search efficiency was decreased by concurrent working memory load. In this case, efficiency in the visual search task could be decreased not because participants had an impaired ability to allocate attention to each item, but because it took relatively longer to process stimuli with a reduced attentional benefit in perception. Given the present results, the participants could have attended the search item despite an active spatial working memory load. However, the perception of each item could not have been improved while the participants attended stimuli because spatial working memory load impaired signal enhancement.

The finding that working memory load did not impair allocation of attention seemed inconsistent with the result of Boot et al. (2005), who suggested that working memory load due to a transient event reduced attentional orienting, as measured by reaction time. Consistently with Boot et al., another recent study (Kwon \& Kim, 2004) showed that an executive working memory load (a backward counting task) reduced the effect of an involuntary cue. However, the present study differs from those experiments in several methodological ways. First, the type of working memory task was different. Boot et al. and Kwon and Kim used a secondary task to load the central executive, whereas the present study used a spatial working memory task. Second, in their experiments, the new object onset that captured attention was irrelevant with respect to the target item, but the transient cue used in the present study always predicted the target location. We can say that executive working memory load can disrupt involuntary orienting. In contrast, voluntary orienting by an informative cue was not disrupted by spatial working memory load.

The present experiments show different patterns of interaction depending on type of attentional orienting and working memory. These various patterns of interaction suggest the need for a more concrete model. Voluntary attention could enhance the quality of perceptual representations but involuntary attention would not. Enhanced perceptual processing by voluntary attention would require allocation of mental resources or a top-down feedback signal at the early stage of visual processing (Hamker, 2004). This allocation of mental resources or a reentry signal would be closely related to or share a common resource with spatial working memory processing. Thus, spatial working memory load would reduce the attentional effect of enhanced perception, revealed mainly by the benefit in accuracy found in the present study. In contrast, another type of attentional selection without enhanced perception would be mediated by a separate process from the working memory store. On the other hand, working memory load could disrupt involuntary orienting by a transient event because detecting and processing a task-irrelevant visual event was interfered with by maintaining visual information actively in working memory. Another important point is that the interaction between working memory and attention could differ not only according to the type of attention but also according to the type of working memory task.

A recent fMRI brain imaging study (Postle et al., 2004) agrees with the finding of the present study that spatial working memory load does not disrupt every type of attentional orienting. According to Postle et al., dissociation could occur between brain activation areas during attention tasks and during spatial working memory tasks, but the two activation areas showed considerable overlap, consistent with previous imaging studies (Awh et al., 1999; Corbetta et al., 2002) and the behavioral study (Awh et al., 1998). However, using event-related fMRI, Postle et al. reported that the frontal eye field activated during a selective attention task was not activated when spatial information was maintained. Given that the frontal eye field is critical in attentional orienting during spatial cuing and visual search (Hamker, 2004; Serences \& Yantis, 2006), this result can be linked to present behavioral data to show a different pattern of interaction between spatial working memory and attentional orienting depending on the type of attentional processing.

The present study also showed that cognitive processing for the maintenance of spatial information influences visual processing in the perceptual stage. This is also consistent with Awh et al. (1999), who reported that spatial working memory affected early sensory modulation. Furthermore, according to a recent behavioral study (Lee \& Vecera, 2005), visual short-term memory load could disrupt amodal completion, which has shown that visual working memory influences early vision processing. Furthermore, Jonides et al. (2005) suggested that working memory storage would be mediated by a mechanism for processing perceptual information. In a recent review, Slotnick (2004) proposed that common neural substrates could mediate visual memory and perception. Our data showed that working memory affected visual perception through interaction with attentional processing. This also suggests the possibility that the limited perceptual resources used to enhance the sensory signal of an attended location would be recruited to maintain spatial information in working memory. Since the visual system is a highly interactive network with numerous feedforward and feedback signals (Hamker, 2004; Hockstein \& Ahissar, 2002; Serences \& Yantis, 2006), the interaction between cognitive processing and low-level perception should be further investigated.

The main findings of the present study can be summarized as follows. Spatial working memory load did not change the magnitude of the attentional cuing effect, as measured by reaction time, but it did decrease the cuing effect as measured by accuracy. This result suggests that spatial working memory load does not influence channel selection, but rather that it impairs channel enhancement. In addition, spatial working memory load was not found to disrupt attentional orienting, which implies that the maintenance of spatial information and attentional orienting could be mediated by independent and separate mechanisms. Finally, working memory can influence visual processing in the perceptual stage by interacting with attention. 


\section{AUTHOR NOTE}

The present research was supported by Grant M103KV01001706K2201-01710 from the Brain Research Center of the 21st Century Frontier Research Program, funded by the Ministry of Science and Technology, Republic of Korea. S.W.H. is now in the Department of Psychology at Vanderbilt University. Correspondence concerning this article should be addressed to M.-S. Kim, Department of Psychology, Yonsei University, Seoul, 120-749, Korea (e-mail: kimm@yonsei.ac.kr).

\section{REFERENCES}

AwH, E., \& JonIDES, J. (2001). Overlapping mechanisms of attention and spatial working memory. Trends in Cognitive Sciences, 5, 119-126.

Awh, E., Jonides, J., \& Reuter-Lorenz, P. A. (1998). Rehearsal in spatial working memory. Journal of Experimental Psychology: Human Perception \& Performance, 24, 780-790.

Awh, E., Jonides, J., Smith, E. E., Buxton, R. B., Frank, L. R., Love, T., ET AL. (1999). Rehearsal in spatial working memory: Evidence from brain imaging. Psychological Science, 10, 433-437.

Boot, W. R., Brockmole, J. R., \& Simons, D. J. (2005). Attention capture is modulated in dual-task situations. Psychonomic Bulletin \& Review, 12, 662-668.

Brainard, D. H. (1997). The Psychophysics Toolbox. Spatial Vision, 10, 433-436.

Carrasco, M., Williams, P. E., \& Yeshurun, Y. (2002). Covert attention increases spatial resolution with or without masks: Support for signal enhancement. Journal of Vision, 2, 467-479.

Corbetta, M., Kincade, J. M., \& Shulman, G. L. (2002). Neural systems for visual orienting and their relationship to spatial working memory. Journal of Cognitive Neuroscience, 14, 308-323.

Cowan, N. (2001). The magical number 4 in short-term memory: A reconsideration of mental storage capacity. Behavioral \& Brain Sciences, 24, 87-185.

ENGLE, R. W. (2002). Working memory capacity as executive attention. Current Directions in Psychological Science, 11, 19-23.

HAMKER, F. (2004). A dynamic model of how feature cues guide spatial attention. Vision Research, 44, 501-521.

Hockstein, S., \& Ahissar, M. (2002). View from the top: Hierarchies and reverse hierarchies in the visual system. Neuron, 36, 791-804.

JoNIDES, J. (1981). Voluntary versus automatic control over the mind's eye's movement. In J. Long \& A. Baddeley (Eds.), Attention and performance IX (pp. 187-203). Hillsdale, NJ: Erlbaum.

Jonides, J., LACEY, S. C., \& NeE, D. E. (2005). Processes of working memory in mind and brain. Current Directions in Psychological Science, 6, 376-378.

KwON, M. Y., \& KIM, M.-S. (2004). The influence of executive working memory on exogenous and endogenous cuing effects. Korean Journal of Experimental Psychology, 16, 451-466.

LEE, H., \& VeCERA, S. P. (2005). Visual cognition influences early vision. Psychological Science, 16, 763-768.

Lu, Z.-L., \& Dosher, B. A. (1998). External noise distinguishes attention mechanisms. Vision Research, 38, 1183-1198.

Luck, S. J., Hillyard, S. A., Mouloua, M., \& Hawkins, H. L. (1996). Mechanisms of visual-spatial attention: Resource allocation or un- certainty reduction? Journal of Experimental Psychology: Human Perception \& Performance, 22, 725-737.

LUCK, S. J., \& Thomas, S. J. (1999). What variety of attention is automatically captured by peripheral cues? Perception \& Psychophysics, 61, 1424-1435.

MayfranK, L., Kimmig, H., \& Fischer, B. (1987). The role of attention in the preparation of visually guided saccadic eye movements in man. In J. K. O'Regan \& A. Levy-Schoen (Eds.), Eye movements: From physiology to cognition (pp. 37-45). New York: North-Holland.

Moore, C. M., \& Egeth, H. (1998). How does feature-based attention affect visual processing? Journal of Experimental Psychology: Human Perception \& Performance, 24, 1296-1310.

Mordkoff, J. T., \& Egeth, H. E. (1993). Response time and accuracy revisited: Converging support for the interactive race model. Journal of Experimental Psychology: Human Perception \& Performance, 19, 981-991.

Norman, D. A., \& Bobrow, D. G. (1975). On data-limited and resourcelimited processes. Cognitive Psychology, 4, 44-64.

OH, S.-H., \& KIM, M.-S. (2004). The role of spatial working memory in visual search efficiency. Psychonomic Bulletin \& Review, 11, 275-281.

Pearson, D. G., \& Sahraie, A. (2003). Oculomotor control and the maintenance of spatially and temporally distributed events in visuospatial working memory. Quarterly Journal of Experimental Psychology, 56A, 1089-1111.

Postle, B. R., Awh, E., Jonides, J., Smith, E. E., \& D’Esposito, M. (2004). The where and how of attention-based rehearsal in spatial working memory. Cognitive Brain Research, 20, 194-205.

Prinzmetal, W., McCool, C., \& PARK, S. (2005). Attention: Reaction time and accuracy reveal different mechanisms. Journal of Experimental Psychology: General, 134, 73-92.

SanteE, J. L., \& Egeth, H. E. (1982). Do reaction time and accuracy measure the same aspect of letter recognition? Journal of Experimental Psychology: Human Perception \& Performance, 8, 489-501.

Serences, J. T., \& Yantis, S. (2006). Selective visual attention and perceptual coherence. Trends in Cognitive Sciences, 10, 38-45.

Slotnick, S. D. (2004). Visual memory and visual perception recruit common neural substrates. Behavioral \& Cognitive Neuroscience Reviews, 3, 207-221.

Vogel, E. K., Woodman, G. F., \& Luck, S. J. (2001). Storage of features, conjunctions, and objects in visual working memory. Journal of Experimental Psychology: Human Perception \& Performance, 27, 92-114.

Woodman, G. F., \& LucK, S. J. (2004). Visual search is slowed when visuospatial working memory is occupied. Psychonomic Bulletin \& Review, 11, 269-274.

Woodman, G. F., Vogel, E. K., \& Luck, S. J. (2001). Visual search remains efficient when visual working memory is full. Psychological Science, 12, 219-224.

Yeshurun, Y., \& CARRAsco, M. (1999). Spatial attention improves performance in spatial resolution tasks. Vision Research, 39, 293-306.

(Manuscript received July 19, 2006; revision accepted for publication December 1, 2007.) 